\title{
SOME AUTOMORPHISM GROUPS IN NOETHERIAN DOMAINS
}

\author{
BY J. NEGGERS
}

Communicated by David A. Buchsbaum, October 19, 1967

Suppose $R$ is a Noetherian domain. Then a quasi-multiplication $f: R \rightarrow R$ is a function $f$ such that $f(x) \in(x)$, for all $x \in R$. A function $f: R \rightarrow R$ such that $\partial f(a, x)=f(a+x)-f(a)$ is a quasi-multiplication, is $q$-differentiable at $a$. In [1] we studied quasi-multiplications on general $R$-modules, $R$ commutative. In particular, we obtained some criteria for a submodule to be an analytic continuation submodule, i.e. a submodule such that the only everywhere differentiable extension of 0 on the submodule to the module is 0 . It is shown (and clear) that an additive map is everywhere $q$-differentiable if and only if it is a quasi-multiplication.

In this paper we show that if $R$ is a Noetherian domain, then the set of everywhere differentiable automorphisms is a normal subgroup of the group of automorphisms of $R$. With these automorphisms one can duplicate to a certain extent the constructions made with analytic automorphisms on complete discrete valuation rings. In particular, one can define analogues of the pseudo-ramification groups and the groups of strongly inertial automorphisms. We show that in a Noetherian domain every nonzero ideal is an analytic continuation ideal relative to the everywhere $q$-differentiable automorphisms, i.e. every automorphism of a nonzero ideal which is everywhere $q$-differentiable has a unique extension to an everywhere $q$-differentiable automorphism on the entire domain. As a consequence we see that if the ideal is an analytic continuation ideal, then these automorphisms can only be extended to automorphisms if we permit extensions out of the collection of everywhere $q$-differentiable maps on $R$.

The Groups $G_{R}, G_{\{\bar{a}\rangle}, G_{\langle\bar{a}\rangle}, G_{\bar{a}}$. If $R$ is a Noetherian domain and if $a \neq(0)$ is an ideal, then we define the following sets:

$$
\begin{aligned}
G_{R} & =\{\sigma \mid \sigma \text { is an automorphism and a quasi-multiplication }\}, \\
G_{\{\bar{a}\}} & =\left\{\sigma \in G_{R} \mid \sigma(x)-x \in \bar{a} \text { for all } x \in R\right\}, \\
G_{\langle\bar{a}\rangle} & =\left\{\sigma \in G_{R} \mid \sigma(x)=u(x) x, u(x) \in 1+\bar{a} \text { for all } x \in R\right\} .
\end{aligned}
$$

The set $G_{\bar{a}}$ is defined as $G_{R}$.

THEOREM 1. If $G$ is the group of automorphisms on $R$, then

$$
G_{R} \triangleleft G, \quad G_{\{a\}} \triangleleft G_{R}, \quad G\langle\triangleleft\rangle \triangleleft G_{R} .
$$


Proof. Suppose $\sigma(x)=u(x) x$. Then since $\sigma(x) \in(x)$, we have a chain of ideals

$$
(x) \subset\left(\sigma^{-1}(x)\right) \subset \cdots \subset\left(\sigma^{-n}(x)\right) \subset \cdots
$$

The ascending chain condition gives an integer $n$ such that $\left(\sigma^{-n}(x)\right)$ $=\left(\sigma^{-n-1}(x)\right)$. Let $y=\sigma^{-n-1}(x)$. Then $(y)=(\sigma(y))=\sigma((y))$ and $(y)$ $=\sigma^{m}(y)$ for all $m$. Thus, $m=n+1, n+2$ implies $(x)=\sigma((x))=(\sigma(x))$. If we let $x=\rho(x) \sigma(x), \sigma^{-1}(x)=\sigma^{-1}((x)) x=v(x) x$ and $G_{R}$ is a group.

Since $(x)=(\sigma(x))=(u(x) x)$, we have $\rho(x) u(x)=1$ and $u(x)$ is a unit $(u(0)=1)$, while $v(x)=\sigma^{-1}\left([u(x)]^{-1}\right)=\left[u\left(\sigma^{-1}(x)\right)\right]^{-1}$, and $\sigma^{-1}(u(x))$ $=u\left(\sigma^{-1}(x)\right)$.

Now suppose $\tau \in G, \sigma \in G_{R}$. Since $\tau(1)=1, \tau$ maps units onto units. Hence, $\tau \sigma \tau^{-1}(x)=\tau\left(u\left(\tau^{-1}(x)\right) \tau^{-1}(x)\right)=\tau u \tau^{-1}(x) \cdot x$, and since $u \tau^{-1}(x)$ is a unit, $\tau u \tau^{-1}(x)$ is a unit. Thus, $G_{R} \triangleleft G$.

If $\sigma \in G_{\{a\}}$, then $\sigma\left(\sigma^{-1}(x)\right)-\sigma^{-1}(x) \in \bar{a}$ and $\sigma^{-1} \in G_{\{a\}}$. Furthermore, if $\tau \in G_{R}, \tau(a)=\bar{a}$ and, hence, $\tau \sigma \tau^{-1}(x)-x=\tau\left\{\sigma\left(\tau^{-1}(x)\right)-\tau^{-1}(x)\right\} \in \bar{a}$ and $G_{\{a \mid} \triangleleft G_{R}$.

If $\sigma \in G_{\langle a\rangle}$, we write $u(x)=1+a(x)$. Then $v(x)=\left[1+a\left(\sigma^{-1}(x)\right)\right]^{-1}$ and $v(x)-1=-a\left(\sigma^{-1}(x)\right)\left[1+a\left(\sigma^{-1}(x)\right)\right]^{-1}$, and so $v(x) \in 1+\bar{a}$, i.e. $\sigma^{-1} \in G_{\langle(a)}$. Similarly, if $\tau \in G_{R}$, then $\tau u \tau^{-1}(x)=1+\tau\left(a\left(\tau^{-1}(x)\right)=1\right.$ $+v\left(a\left(\tau^{-1}(x)\right) a\left(\tau^{-1}(x)\right) \in 1+\bar{a}\right.$, and $G_{\langle a|} \in G_{R}$, which concludes the proof.

Now, if $\sigma \in G_{R}$, then $\sigma(\bar{a})=\bar{a}$ and, hence, $\left.\sigma\right|_{a} \in G_{d}$. We have a mapping $\phi$ defined by $\phi(\sigma)=\left.\sigma\right|_{a}$. Clearly, $\phi$ is a homomorphism.

TheOREM 2. The mapping $\phi$ is an isomorphism $\phi: G_{R} \rightarrow G_{a}$.

Proof. If $\lambda \in R$, then $\sigma(\lambda a)=\sigma(\lambda) \sigma(a)$, and hence if $\left.\sigma\right|_{a}$ is the identity, $\sigma(\lambda a)=\lambda a=\sigma(\lambda) a$, whence $\sigma(\lambda)=\lambda$.

Now, if $\sigma \in G_{a}$, define $\sigma: R \rightarrow R$ by $\sigma(\lambda)=u(\lambda a)[u(a)]^{-1} \lambda$.

Since $\sigma\left(\lambda a a^{\prime}\right) / \sigma\left(\lambda a a^{\prime}\right)=1=u(\lambda a) u\left(a^{\prime}\right) / u\left(\lambda a^{\prime}\right) u(a)$, it follows that the definition of $\sigma$ is independent of the choice of $a \in \bar{a}$, as long as $a \neq 0$. Now $\sigma\left(\lambda_{1}+\lambda_{2}\right)=u\left(\lambda_{1} a+\lambda_{2} a\right)[u(a)]^{-1}\left(\lambda_{1}+\lambda_{2}\right)$. Computing $u\left(\lambda_{1} a+\lambda_{2} a\right)$ from $\sigma\left(\lambda_{1} a+\lambda_{2} a\right)$, we find $u\left(\lambda_{1} a+\lambda_{2} a\right)=\left(u\left(\lambda_{1} a\right) \lambda_{1}\right.$ $\left.+u\left(\lambda_{2} a\right) \lambda_{2}\right)\left(\lambda_{1}+\lambda_{2}\right)$, and thus $\sigma\left(\lambda_{1}+\lambda_{2}\right)=\sigma\left(\lambda_{1}\right)+\sigma\left(\lambda_{2}\right)$. Similarly, $\sigma\left(\lambda_{1} \lambda_{2}\right)=u\left(\lambda_{1} \lambda_{2} a^{2}\right)\left[u\left(a^{2}\right)\right]^{-1} \lambda_{1} \lambda_{2}=u\left(\lambda_{1} a\right) u\left(\lambda_{2} a\right)[u(a)]^{-2} \lambda_{1} \lambda_{2}=\sigma\left(\lambda_{1}\right) \sigma\left(\lambda_{2}\right)$.

Now let $\sigma^{-1} \in G_{a}$ be the inverse of $\sigma$ on $\bar{a}$. Then $\sigma^{-1}(\lambda)=v(\lambda a)$ - $[v(a)]^{-1} \lambda$ is the extension of $\sigma^{-1}$ to $R$. Then $\sigma\left(\sigma^{-1}(\lambda)\right)=u\left(\sigma^{-1}(\lambda) a\right)$ $\cdot[u(a)]^{-1} \sigma^{-1}(\lambda)=u\left(\sigma^{-1}(\lambda) a\right)[u(a)]^{-1} v(\lambda a)[v(a)]^{-1} \lambda$.

We assert that

$$
u\left(\sigma^{-1}(\lambda) a\right)[u(a)]^{-1} v(\lambda a)[v(a)]^{-1}=1,
$$

whence $\sigma\left(\sigma^{-1}(\lambda)\right)=\lambda$ and $\sigma \in G_{R}$. 
A computation yields

$$
\begin{aligned}
& u\left(\sigma^{-1}(\lambda) a^{2}\right)\left[u\left(a^{2}\right)\right]^{-1} v(\lambda a)^{2}\left[v\left(a^{2}\right)\right]^{-1} \\
& \quad=u\left(\sigma^{-1}\left(\lambda a^{2}\right)\left[1-\left(a^{2}\right)\right]^{-1}\right) \sigma^{-1}\left(\lambda a^{2}\right)\left[v\left(a^{2}\right)\right]^{-1}\left[\sigma\left(a^{2}\right)\right]^{-1} \lambda^{-1} \\
& \quad=\sigma\left(\sigma^{-1}\left(\lambda a^{2}\right)\left[1-\left(a^{2}\right)\right]^{-1}\right)\left[\sigma\left(a^{2}\right)\right]^{-1} \lambda^{-1} \\
& \quad=\lambda a \sigma\left(\sigma^{-1}(a)\left[v\left(a^{2}\right)\right]^{-1}\right)\left[\sigma\left(a^{2}\right)\right]^{-1} \lambda^{-1} \\
& =\sigma\left(\sigma^{-1}(a)\left[v\left(a^{2}\right)\right]^{-1}\right)\left[\sigma\left(a^{2}\right)\right]^{-1} a \\
& =\sigma\left(\left[v\left(a^{2}\right)\right]^{-1}\right)\left[u\left(a^{2}\right)\right]^{-1} \\
& =\sigma\left(u\left(\sigma^{-1}\left(a^{2}\right)\right) \sigma\left(\sigma^{-1}\left(a^{2}\right)\right)\left[\sigma\left(a^{2}\right)\right]^{-1}\right. \\
& =\sigma\left(u\left(\sigma^{-1}\left(a^{2}\right)\right) \sigma^{-1}\left(a^{2}\right)\right)\left[\sigma\left(a^{2}\right)\right]^{-1} \\
& =\sigma\left(a^{2}\right)\left[\sigma\left(a^{2}\right)\right]^{-1} \\
& =1
\end{aligned}
$$

and the theorem follows.

CoROllary 1. If $b^{\prime} \subset R$ is a nonzero ideal, then $u: b^{\prime} \rightarrow 1+\bar{a}$ implies $u: R \rightarrow 1+\bar{a}$.

Proof. For $b \in b^{\prime}$, we write $u(b)=1+a(b)$. Then $u(\lambda) u(b)=u(\lambda b)$ $=1+a(\lambda b)$ and $u(\lambda)[u(b)-1]+(u(\lambda)-1)=u(\lambda b)-1=a(\lambda b)$, whence $u(\lambda)-1=a(\lambda b)-u(\lambda) a(b) \in \bar{a}$.

Corollary 2. Given a Noetherian domain $R$, an ideal $\bar{a} \subset R, \bar{a} \neq(0)$ is an analytic continuation ideal if and only if some automorphism $\sigma \in G_{a}$ has a unique, everywhere differentiable extension to an automorphism $\sigma \in G_{R}$. If it is an analytic continuation ideal, every $\sigma \in G_{a}$ has such a unique extension.

REMARKs. In the case $R$ is a complete discrete valuation ring, $G_{R}=G, G_{\left\{(\pi)^{n}\right\}}=G_{n}$, and $G_{\left\langle(\pi)^{n}\right\rangle}=\bar{G}_{n}$. Also, $R$ has no analytic continuation ideals since $(\pi)$ is not an analytic continuation ideal. This is so because $\delta$, given by $\delta(x)=\{0$ if $x \in(\pi), x$ if $x \notin(\pi)\}$, is everywhere $q$-differentiable. On the other hand, using a technique similar to the proof of Theorem 9 [1], one shows that every nonzero ideal in $Z$ is an analytic continuation ideal. If $\not \subset R$ is a prime ideal, there is an extension map $\phi: G_{R} \rightarrow G_{R}$, which is a monomorphism. This map induces monomorphisms $G_{\{\bar{a}\}} \rightarrow G_{\left\{\overline{a_{\bar{p}}}\right\rangle}, G_{\langle\bar{a}\rangle} \rightarrow G_{\langle a \bar{p}\rangle}$, where in the latter case if $\bigcap_{n}\left(\tilde{a}_{\bar{p}}\right)^{n}=(0)$, we may write

$$
a\left(\frac{x}{y}\right)=a(x)+\sum_{i=0}^{\infty}(-1)^{i} u(x) a(y)^{i},
$$


the convergence being a consequence of the existence of the injection $G_{\langle\bar{a}\rangle} \rightarrow G_{\left\langle\bar{a}_{\bar{p}}\right\rangle}$

For $\sigma \in G_{\{a\}}$ and $\bar{a}=(a)$ a principal ideal, it follows that if $\sigma(x)$ $=x+a \cdot A(x), A$ induces a derivation on $R /(a)$. In particular, if $R /(a)$ has no nontrivial derivations $G_{\{a\}}=G_{\left\{a^{2}\right\}}$ and hence by induction $G_{\{a\}}=G_{\left\{a^{m}\right\}}$ for all $m$. Hence, if $\cap \bar{a}^{n}=(0), G_{\{\bar{a}\}}=G_{\{0\}}=1$, the identity map, and $G_{\left\{b^{\prime}\right\}}=1$ for all ideals $b^{\prime} \subset \bar{a}$. The group $G_{\{\bar{a}\}}$ is the kernel of the map $G_{R} \rightarrow G_{R / \tilde{d}}$.

\section{REFERENCE}

1. J. Neggers, Inertial mappings and differentiation on R-modules, (to appear).

The University of Alabama 